\title{
Biomarkers of Oncogenesis, Adipose Tissue Dysfunction and Systemic Inflammation for the Detection of Hepatocellular Carcinoma in Patients with Nonalcoholic Fatty Liver Disease
}

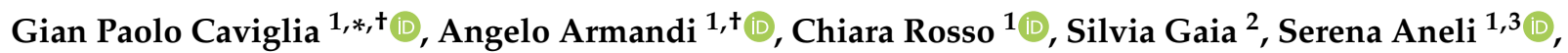 \\ Emanuela Rolle ${ }^{2}$, Maria Lorena Abate ${ }^{1}{ }^{1}$, Antonella Olivero ${ }^{1}{ }^{1}$, Aurora Nicolosi ${ }^{1}$, Marta Guariglia ${ }^{1}{ }^{(0}$, \\ Davide Giuseppe Ribaldone ${ }^{1}\left(\mathbb{D}\right.$, Patrizia Carucci ${ }^{2}$, Giorgio Maria Saracco ${ }^{1,2}{ }^{(1)}$ and Elisabetta Bugianesi ${ }^{1,2, *}$
}

check for updates

Citation: Caviglia, G.P.; Armandi, A. Rosso, C.; Gaia, S.; Aneli, S.; Rolle, E.; Abate, M.L.; Olivero, A.; Nicolosi, A.; Guariglia, M.; et al. Biomarkers of Oncogenesis, Adipose Tissue Dysfunction and Systemic

Inflammation for the Detection of Hepatocellular Carcinoma in Patients with Nonalcoholic Fatty Liver Disease. Cancers 2021, 13, 2305. https://doi.org/10.3390/ cancers13102305

Academic Editor: Atsushi Umemura

Received: 9 April 2021

Accepted: 7 May 2021

Published: 11 May 2021

Publisher's Note: MDPI stays neutral with regard to jurisdictional claims in published maps and institutional affiliations.

Copyright: (c) 2021 by the authors. Licensee MDPI, Basel, Switzerland. This article is an open access article distributed under the terms and conditions of the Creative Commons Attribution (CC BY) license (https:/ / creativecommons.org/licenses/by/ $4.0 /)$.
1 Department of Medical Sciences, University of Turin, 10126 Torino, Italy; angelo.armandi@unito.it (A.A.); chiara.rosso@unito.it (C.R.); serena.aneli@unito.it (S.A.); marialorena.abate@unito.it (M.L.A.); antonella.olivero@unito.it (A.O.); aurora.nicolosi999@edu.unito.it (A.N.); marta.guariglia@edu.unito.it (M.G.); davidegiuseppe.ribaldone@unito.it (D.G.R.); giorgiomaria.saracco@unito.it (G.M.S.)

2 Gastroenterology Unit, A.O.U. Città della Salute e della Scienza, 10126 Torino, Italy; sgaia2@cittadellasalute.to.it (S.G.); erolle@cittadellasalute.to.it (E.R.); pcarucci@cittadellasalute.to.it (P.C.)

3 Department of Biology, University of Padua, 35122 Padova, Italy

* Correspondence: gianpaolo.caviglia@unito.it (G.P.C.); elisabetta.bugianesi@unito.it (E.B.); Tel.: +39-11-633-3532 (G.P.C.)

+ Co-first authors.

Simple Summary: Circulating biomarkers for the detection of hepatocellular carcinoma in patients with dysmetabolic liver disease are an unmet need. In the present study, we observed that serum values of five biomarkers (namely, AFP, PIVKA-II, GPC-3, adiponectin and IL-6) were significantly different between patients with and without hepatocellular carcinoma; the best accuracy for the detection of tumor was achieved by PIVKA-II. Furthermore, we developed a model combining age, gender, PIVKA-II, GPC-3 and adiponectin that showed an excellent performance for the identification of patients with hepatocellular carcinoma. This model may be useful for the surveillance of patients with dysmetabolic liver disease at risk of hepatocellular carcinoma development.

Abstract: Current surveillance strategy for patients with nonalcoholic fatty liver disease (NAFLD) at risk of hepatocellular carcinoma (HCC) development is unsatisfactory. We aimed to investigate the diagnostic accuracy of alpha-fetoprotein (AFP), protein induced by vitamin $\mathrm{K}$ absence or antagonist-II (PIVKA-II), glypican-3 (GPC-3), adiponectin, leptin and interleukin-6 (IL-6), alone or in combination, for the discrimination between NAFLD patients with or without HCC. The biomarkers were investigated in a cohort of 191 NAFLD patients (median age 62, 54-68 years; 121 males and 70 females) with advanced fibrosis/cirrhosis, 72 of whom had a diagnosis of HCC. PIVKA-II showed the best performance for the detection of HCC with an area under the curve (AUC) of 0.853, followed by adiponectin (AUC = 0.770), AFP (AUC = 0.763), GPC-3 (AUC = 0.759) and by IL-6 $(\mathrm{AUC}=0.731$, while the leptin values were not different between patients with and without HCC. The accuracy of the biomarkers' combination was assessed by a stratified cross-validation approach. The combination of age, gender, PIVKA-II, GPC-3 and adiponectin further improved the diagnostic accuracy (AUC $=0.948$ ); the model correctly identified the $87 \%$ of the patients. In conclusion, we developed a model with excellent accuracy for the detection of HCC that may be useful to improve the surveillance of NAFLD patients at risk of tumor development.

Keywords: adiponectin; AFP; GPC-3; HCC; IL-6; leptin; NAFLD; PIVKA-II; surveillance

\section{Introduction}

The epidemiological burden of nonalcoholic fatty liver disease (NAFLD) is rapidly increasing worldwide, with an estimated global prevalence of $25 \%$ in the general population [1]. 
NAFLD includes a broad spectrum of liver diseases, ranging from simple steatosis to nonalcoholic steatohepatitis (NASH), a condition at risk of progression to liver cirrhosis and hepatocellular carcinoma (HCC) [2]. In addition, the coexistence of multiple metabolic risk factors such as obesity and type 2 diabetes mellitus (T2DM) can synergistically promote tumorigenesis in patients with NAFLD and advanced liver disease [3].

Adipose tissue is recognized as endocrine organ able to produce several adipokines, such as adiponectin and leptin, involved in the regulation of metabolism and inflammation [4]. Adipokines dysregulation has been associated with systemic low-grade inflammation [5], impaired hepatocyte proliferation [6] and progression from NASH to HCC [7]. In particular, interleukin-6 (IL-6) can activate the signal transducer and activator of transcription 3 (STAT3) signaling [8], a major intrinsic pathway involved in cell proliferation, migration and survival $[2,9]$.

Currently, surveillance programs for patients at risk for HCC development are based on ultrasound (US) screening at 6-month intervals, while the combined use of serum biomarkers is still a matter of debate [10-13]. However, US screening is limited by the poor sensitivity for the detection of small liver nodules [14,15]; besides, NAFLD-related HCC may develop in the absence of liver cirrhosis [16], hampering the efficacy of current surveillance strategies mainly aimed at patients with cirrhosis. Therefore, novel noninvasive tools able to improve the surveillance of patients with NAFLD are urgently needed.

Among the traditional HCC biomarkers, protein induced by vitamin $\mathrm{K}$ absence or antagonist II (PIVKA-II), alone or in combination with alpha-fetoprotein (AFP), showed promising results for the early detection, and even for the prediction, of HCC in patients with cirrhosis of viral etiology [17-20]. However, the data on NAFLD-related HCC are scant. Glypican-3 (GPC-3) is an oncofetal protein normally not expressed in the livers of healthy adults; so far, the available data on the performance of serum GPC-3 for HCC detection are conflicting and mainly confined to patients chronically infected with the hepatitis B virus (HBV) or hepatitis $C$ virus (HCV) [21].

To date, the early detection of HCC represents the major goal in order to improve patients' survival; thus, the identification of high-performing biomarkers able to promptly identify patients with HCC among patients at risk of tumor development is an unmet need. Here, we investigated the diagnostic accuracy of selected tumor biomarkers (i.e., AFP, PIVKA-II and GPC-3); adipokines (i.e., adiponectin and leptin) and IL-6, alone or in combination, for the discrimination between patients with or without NAFLD-related HCC.

\section{Materials and Methods}

\subsection{Patients}

This retrospective case-control study included patients with dysmetabolic inducedHCC and patients with NAFLD/NASH without HCC, recruited at the outpatient clinic of the Unit of Gastroenterology of A.O.U. Città della Salute e della Scienza di TorinoMolinette Hospital, Turin, Italy between November 2012 and January 2020.

For all patients, the inclusion criteria were age $\geq 18$ years, histological diagnosis of NASH with advanced fibrosis/cirrhosis or clinical/radiological evidence of cryptogenic cirrhosis [22], which dysmetabolic etiology was assessed by the presence of metabolic risk factors (central obesity, T2DM, dyslipidemia and hypertension) [23] in the absence of other known causes of liver damage. For patients without HCC, a minimum of 1-year US follow-up after the collection of the serum sample was required. All patients signed written informed consent.

We excluded patients with a liver disease of other etiology, such as drug-induced liver disease, viral hepatitis and autoimmune, cholestatic and metabolic/genetic liver disease. Alcohol-induced liver disease was excluded by selecting patients with a negative history of alcohol abuse (weekly ethanol consumption $<140 \mathrm{~g}$ for women and $<210 \mathrm{~g}$ for men) [24].

The presence of advanced fibrosis/cirrhosis was histologically assessed and scored as described by Kleiner et al. [25] or clinically determined by means of a liver elastography (FibroScan ${ }^{\circledR}$, Echosens $^{\mathrm{TM}}$, Paris, France) or hepatic US features and endoscopic signs of 
portal hypertension [26,27]. The diagnosis of HCC was achieved by histological examination or by contrast-enhanced imaging methods showing the radiological hallmark of HCC (i.e., the combination of hypervascularity in the late arterial phase and washout on portal venous and/or delayed phases), following the international guidelines [11]. HCC was classified according to the BCLC staging system $(0=$ very early, $\mathrm{A}=$ early, $\mathrm{B}=$ intermediate, $\mathrm{C}=$ advanced and $\mathrm{D}=$ terminal stage) [11].

\subsection{Measurement of Circulating Biomarkers}

Serum and plasma samples were collected in polypropylene 2-mL tubes labeled with the study participant identification code and stored at $-80^{\circ} \mathrm{C}$ until analysis. Serum levels of AFP, PIVKA-II, adiponectin and IL-6 were determined on the fully automated chemiluminescent enzyme immunoassay (CLEIA) system, LUMIPULSE G600 II analyzer (Fujirebio Inc., Tokyo, Japan) using Lumipulse ${ }^{\circledR}$ G AFP-N (assay precision $<3 \%$ ), Lumipulse ${ }^{\circledR} \mathrm{G}$ PIVKA-II (assay precision $<4.4 \%$ ), Lumipulse ${ }^{\circledR}$ G HMW Adiponectin (assay precision $\leq 4 \%$ ) and IL-6 LPG reaction cartridges, according to the manufacturer's instructions. The lower limit of detection was $0.075 \mathrm{ng} / \mathrm{mL}$ for AFP, $1.37 \mathrm{mAU} / \mathrm{mL}$ for PIVKA-II, $0.09 \mu \mathrm{g} / \mathrm{mL}$ for adiponectin and $0.2 \mathrm{pg} / \mathrm{mL}$ for IL-6.

Serum GPC-3 and plasma leptin values were measured by enzyme-linked immunosorbent assay (ELISA) using CanAg Glypican-3 EIA (Fujirebio Diagnostics AB, Gothenburg, Sweden) and Human Leptin Quantikine ${ }^{\circledR}$ ELISA (R\&D Systems, Minneapolis, MN, USA), according to the manufacturer's instruction. The GPC-3 serum levels were reported in $\mathrm{pg} / \mathrm{mL}$, while the leptin plasma levels were reported in $\mathrm{ng} / \mathrm{mL}$.

\subsection{Statistical Analysis}

Continuous variables were expressed as median and interquartile ranges (IQR), while categorical variables as number and percentages (\%). The $\mathrm{D}^{\prime}$ Agostino-Pearson test was used to test the data normality. The Mann-Whitney test and Kruskal-Wallis test were used to compare continuous variables between two or more groups, respectively. The correlation between continuous variables was assessed by Spearman's rank correlation coefficient $\left(r_{s}\right)$. To evaluate the diagnostic accuracy of the circulating AFP, PIVKA-II, GPC-3, adiponectin, leptin and IL-6 alone, the AUC was assessed by receiver operating characteristic (ROC) curves analysis. A multivariate logistic regression analysis was performed to combine independent variables for the prediction of HCC. This analysis has been repeated using a cross-validation approach to compute its performances in predicting the HCC status, using the scikit-learn package in the Python environment. Specifically, the RepeatedStratifiedKFold with 5 splits and shuffling samples 20 times and the LogisticRegression functions were used to evaluate the performance of the classifier using a cross-validation approach, both with the option random_state $=0$. The final performance of the model were computed, averaging the AUC values over the 100 test sets obtained with the above-described crossvalidated approach. Confidence intervals at a $95 \%$ confidence level were computed with a bootstrap approach by resampling with a replacement 1000 times.

A two-tailed $p<0.05$ was considered statistically significant. The statistical analyses were performed using MedCalc software, version 18.9.1 (MedCalc bvba, Ostend, Belgium) and in-house scripts in Python programming language.

\section{Results}

\subsection{Patients' Characteristics}

A total of 191 patients (median age 62, 54-68 years; male $(\mathrm{M})=121$ and female $(\mathrm{F})=70)$ were included in the study. The demographic, clinical and biochemical characteristics of the study population are reported in Table 1. 
Table 1. Characteristics of the patients included in the study according to the diagnosis of HCC.

\begin{tabular}{|c|c|c|c|}
\hline Characteristics & Controls & HCC & $p$-Value \\
\hline Patients, $n$ & 119 & 72 & \\
\hline Age (years), median (IQR) & $58(49-66)$ & $67(62-70)$ & $<0.001$ \\
\hline Male gender, $n(\%)$ & $64(54 \%)$ & $57(79 \%)$ & $<0.001$ \\
\hline BMI $\left(\mathrm{kg} / \mathrm{m}^{2}\right)$, median (IQR) & $30.8(28.0-34.0)$ & $30.4(26.0-31.7)$ & 0.013 \\
\hline Obesity $\left(\mathrm{BMI} \geq 30.0 \mathrm{~kg} / \mathrm{m}^{2}\right), n(\%)$ & $72(61 \%)$ & $39(54 \%)$ & 0.450 \\
\hline T2DM, $n(\%)$ & $68(57 \%)$ & $43(60 \%)$ & 0.764 \\
\hline Dyslipidemia, $n(\%)$ * & $41(57 \%)$ & $83(69 \%)$ & 0.086 \\
\hline Hypertension, $n(\%)$ & $88(74 \%)$ & $54(75 \%)$ & 1.000 \\
\hline $\operatorname{ALT}(\mathrm{U} / \mathrm{L})$, median (IQR) & $53(31-72)$ & $34(24-41)$ & $<0.001$ \\
\hline AST (U/L), median (IQR) & $41(30-57)$ & $38(30-50)$ & 0.280 \\
\hline$\gamma \mathrm{GT}(\mathrm{U} / \mathrm{L})$, median (IQR) & $63(43-120)$ & 87 (57-179) & 0.023 \\
\hline Platelets $\left(\times 10^{9} / \mathrm{L}\right)$, median $(\mathrm{IQR})$ & $194(161-239)$ & $132(85-183)$ & $<0.001$ \\
\hline Albumin (g/dL), median (IQR) & $4.1(3.8-4.4)$ & $3.9(3.3-4.1)$ & $<0.001$ \\
\hline Total Bilirubin (mg/dL), median (IQR) & $0.8(0.6-1.0)$ & $0.9(0.7-1.5)$ & 0.001 \\
\hline INR, median (IQR) & $1.05(1.00-1.11)$ & $1.14(1.09-1.31)$ & $<0.001$ \\
\hline Total Cholesterol (mg/dL), median (IQR) & $175(157-203)$ & $168(139-181)$ & 0.025 \\
\hline HDL-Cholesterol (mg/dL), median (IQR) & $46(39-59)$ & $44(35-54)$ & 0.368 \\
\hline Triglycerides (mg/dL), median (IQR) & $124(94-160)$ & $115(84-132)$ & 0.009 \\
\hline Fasting Glucose (mg/dL), median (IQR) & $110(92-129)$ & $112(100-137)$ & 0.143 \\
\hline Cirrhosis, $n(\%)$ & $66(55 \%)$ & $63(88 \%)$ & $<0.001$ \\
\hline BCLC Stage $(0 / \mathrm{A} / \mathrm{B} / \mathrm{C} / \mathrm{D}), n$ & & $7 / 40 / 15 / 7 / 3$ & \\
\hline HCC nodules $(1 / 2 / 3 />3), n$ & & $33 / 16 / 8 / 15$ & \\
\hline Size of major nodule $(\mathrm{mm})$, median (IQR) & & $18(15-24)$ & \\
\hline
\end{tabular}

* Total cholesterol $\geq 200 \mathrm{mg} / \mathrm{dL}$ and/or HDL cholesterol $<40 \mathrm{mg} / \mathrm{dL}$ for men and $<50 \mathrm{mg} / \mathrm{dL}$ for women and/or triglycerides $\geq 150 \mathrm{mg} / \mathrm{dL}$. $p$-values for the quantitative variables were calculated by Mann-Whitney test, while $p$-values for categorical variables were calculated by Fisher's Exact test. Abbreviations: alanine aminotransferase (ALT), aspartate aminotransferase (AST), Barcelona Clinic Liver Cancer (BCLC), body mass index (BMI), gamma-glutamyl transpeptidase $(\gamma \mathrm{GT})$, hepatocellular carcinoma (HCC), high-density lipoprotein (HDL), international normalized ratio (INR), interquartile range (IQR), low-density lipoprotein (LDL), number $(n)$ and type 2 diabetes mellitus (T2DM).

Patients with HCC $(n=72)$ were older than patients without tumor $(n=119)(67$, $62-70$ years vs. $58,49-66$ years, $p<0.001)$ and had a higher prevalence of males $(n=57$, $79 \%$ vs. $n=64,54 \%, p=0.010)$. No differences were observed in the prevalence of obesity $(p=0.450), \mathrm{T} 2 \mathrm{DM}(p=0.764)$, dyslipidemia $(p=0.086)$ and hypertension $(p=1.000)$. The majority of patients were cirrhotic $(n=129,68 \%)$, with a higher prevalence of cirrhosis in patients with HCC compared to those without tumors ( $88 \%$ vs. $55 \%, p<0.001)$. Consistently, patients with HCC showed significantly higher values of total bilirubin $(p=0.001)$ and INR $(p<0.001)$ and lower values of platelets $(p<0.001)$ and albumin $(p<0.001)$. Among patients without HCC, 95 had a histological diagnosis of NAFLD/NASH (F3 = 53, F4 = 42), while 24 had a clinical diagnosis of cirrhosis. Among the patients with HCC, the diagnosis of tumor was achieved by pathology in 14 patients, by multiphasic computed tomography (CT) in 35 patients and by dynamic contrast-enhanced magnetic resonance imaging (MRI) in 23 patients. Overall, 47 patients had a diagnosis of early tumor (BCLC $0=7, \mathrm{~A}=40$ ) and 22 of advanced tumor (BCLC B $=15, C=7$ ), while three patients had terminal-stage HCC $(\mathrm{BCLC} \mathrm{D}=3)$.

\subsection{Circulating Biomarkers Values in the Study Cohort}

The median serum levels of AFP, PIVKA-II, GPC-3, adiponectin and IL-6 were significantly higher in patients with HCC compared to those without tumor (all $p<0.001$ ); only the plasma leptin values were not different between the two groups of patients $(p=0.649)$ (Table 2 and Figure 1). 
Table 2. Median biomarker levels according to the presence of HCC.

\begin{tabular}{cccc}
\hline Biomarkers & Controls & HCC & $p$-Value \\
\hline AFP $(\mathrm{ng} / \mathrm{mL})$, median IQR & $3.8(2.7-5.1)$ & $6.0(4.5-13.5)$ & $<0.001$ \\
PIVKA-II $(\mathrm{mAU} / \mathrm{mL})$, median IQR & $33(27-45)$ & $121(54-1135)$ & $<0.001$ \\
GPC-3 $(\mathrm{pg} / \mathrm{mL})$, median IQR & $35(20-56)$ & $75(40-104)$ & $<0.001$ \\
Adiponectin $(\mu \mathrm{g} / \mathrm{mL})$, median IQR & $2.17(1.35-3.30)$ & $4.95(2.87-7.03)$ & $<0.001$ \\
Leptin $(\mathrm{ng} / \mathrm{mL})$, median IQR & $20.6(9.8-33.4)$ & $20.3(13.2-34.9)$ & 0.649 \\
IL-6 $(\mathrm{pg} / \mathrm{mL})$, median IQR & $3.1(1.9-5.8)$ & $6.0(4.1-12.5)$ & $<0.001$ \\
\hline
\end{tabular}

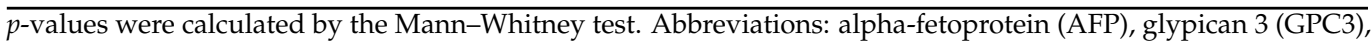
hepatocellular carcinoma (HCC) and interleukin-6 (IL-6).
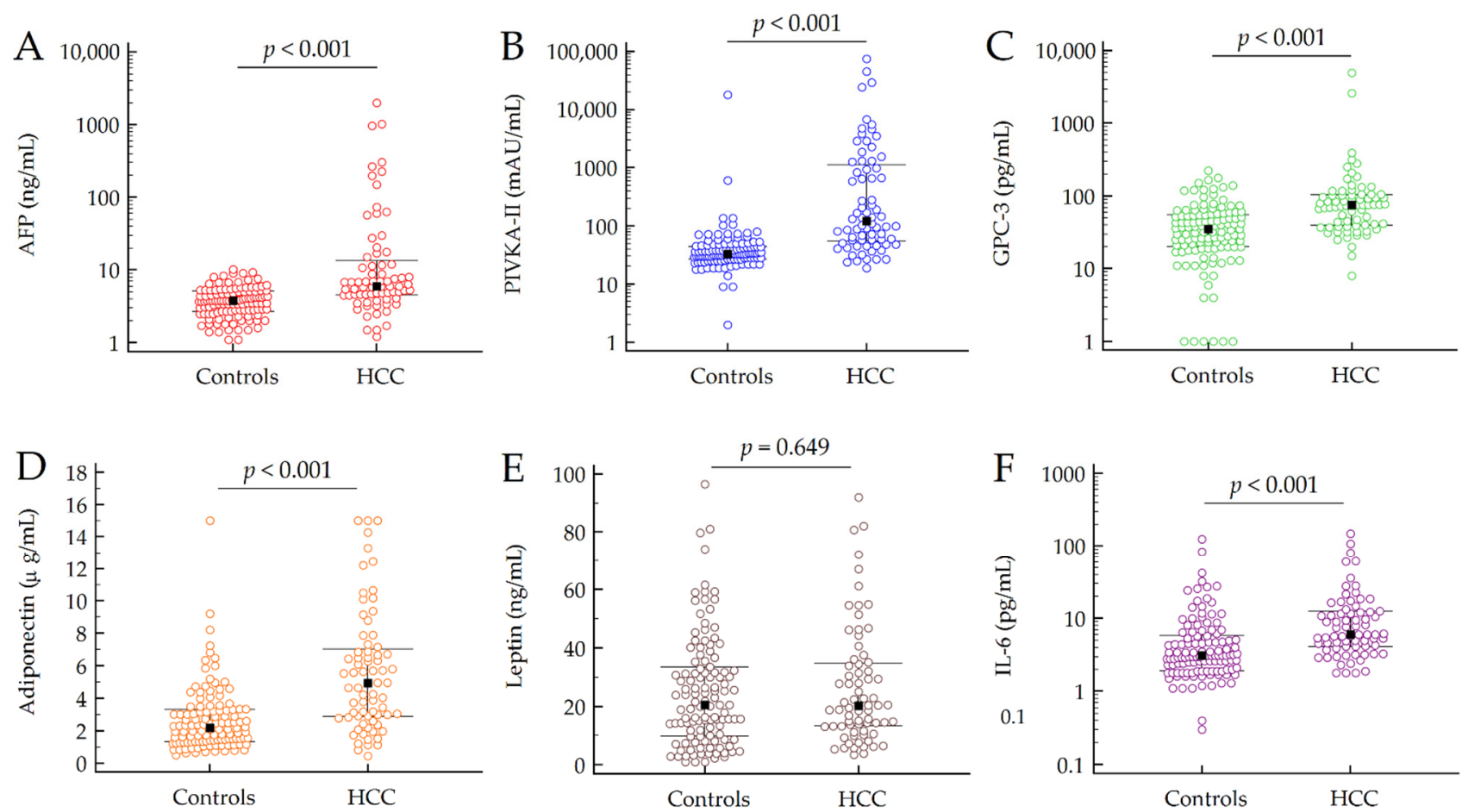

Figure 1. Median values of AFP (A), PIVKA-II (B), GPC-3 (C), adiponectin (D), leptin (E) and IL-6 (F) in patients with and without HCC. $P$-values were calculated by Mann-Whitney test. Black squares and error bars represent, respectively, the median value and the IQR in each group of patients. The values of AFP, PIVKA-II, GPC-3 and IL-6 are depicted in Log scale due to data skewness. Abbreviations: alpha-fetoprotein (AFP), glypican 3 (GPC3), hepatocellular carcinoma (HCC) and interleukin-6 (IL-6).

The AFP serum values showed a significant stepwise increase from patients with advanced fibrosis without HCC to patients with advanced tumor (BCLC $=B, C$ and $D)$ $(p<0.001)$. The AFP and PIVKA-II levels were significantly different between the patients with early HCC $(\mathrm{BCLC}=0, \mathrm{~A})$ and those with advanced tumor $(p<0.050)$, while among patients without HCC, the AFP, GPC3 and IL-6 serum levels were significantly different between the patients with advanced fibrosis and those with cirrhosis $(p<0.050)$ (Table S1 and Figure S1).

By the Spearman correlation analysis, we observed that the serum AFP values were moderately correlated to PIVKA-II $\left(r_{s}=0.408,95 \%\right.$ confidence interval (CI) $0.283-0.520$, $p<0.001)$ and to the GPC-3 serum levels $\left(r_{s}=0.484,95 \%\right.$ CI $\left.0.368-0.586, p<0.001\right)$. PIVKA-II was moderately correlated to GPC-3 $\left(r_{s}=0.311,95 \%\right.$ CI $\left.0.177-0.434, p<0.001\right)$, and GPC-3 was moderately correlated to serum adiponectin $\left(r_{s}=0.304,95 \%\right.$ CI $0.169-0.428$, $p<0.001$ ) (Figure 2). 
1.0

\begin{tabular}{|l|c|c|c|c|c|c|}
\hline AFP & & 0.484 & 0.408 & 0.276 & 0.258 & 0.113 \\
\hline GPC-3 & 0.484 & & 0.311 & 0.207 & 0.304 & 0.129 \\
\hline PIVKA-II & 0.408 & 0.311 & & 0.278 & 0.271 & -0.014 \\
\hline IL-6 & 0.276 & 0.207 & 0.278 & & 0.190 & 0.286 \\
\hline Adiponectin & 0.258 & 0.304 & 0.271 & 0.190 & & 0.154 \\
\hline Leptin & 0.113 & 0.129 & -0.014 & 0.286 & 0.154 & \\
\hline & AFP & GPC-3 & PIVKA-II & IL-6 & Adiponectin & Leptin \\
\hline
\end{tabular}

Figure 2. Correlogram of the biomarkers' concentrations. Cells are colored according to the magnitude of the correlations, ranging from dark red for positive correlations to dark blue for negative correlations. Correlation coefficients $\left(r_{s}\right)$ were calculated by the Spearman test. Abbreviations: alpha-fetoprotein (AFP), glypican 3 (GPC3), hepatocellular carcinoma (HCC) and interleukin-6 (IL-6).

\subsection{Diagnostic Accuracy of Circulating Biomarkers for HCC Detection}

The diagnostic accuracy of AFP, PIVKA-II, GPC-3, adiponectin and IL-6 for the discrimination between patients with and without HCC was assessed by the receiver operating characteristic (ROC) curve analysis. The values of the area under the curve (AUC), sensitivity (Se), specificity (Sp), positive likelihood ratio (+LR) and negative likelihood ratio $(-\mathrm{LR})$ are reported in Table 3. PIVKA-II showed a higher performance with AUC $=0.853$, followed by adiponectin (AUC =0.770), AFP (AUC =0.763), GPC-3 (AUC = 0.759) and by IL-6 (AUC = 0.731) (Figure 3). Similar results were observed, following the stratification for the BMI and T2DM (Figure S2). By ROC curve comparison, the diagnostic accuracy for HCC detection of PIVKA-II was significantly superior to the performance of AFP $(\triangle \mathrm{AUC}=0.090, p=0.044), \mathrm{GPC}-3(\triangle \mathrm{AUC}=0.094, p=0.035)$ and IL-6 $(\triangle \mathrm{AUC}=0.122$, $p=0.009)$, while only a trend was observed for the comparison between PIVKA-II and adiponectin $(\triangle \mathrm{AUC}=0.083, p=0.075)$. No other significant differences were observed from the comparison between AFP, GPC-3, adiponectin and IL-6 (Table S2). Finally, we performed a sub-analysis to investigate the biomarkers accuracy for the detection of early tumors (BCLC $=0$ and A); PIVKA-II showed the higher performance (AUC =0.810), followed by GPC-3 (AUC =0.749), adiponectin $(\mathrm{AUC}=0.744)$, AFP $(\mathrm{AUC}=0.704)$ and by IL-6 (AUC = 0.699) (Figure S3).

Table 3. Diagnostic accuracy of AFP, PIVKA-II, GPC-3, adiponectin and IL-6 discrimination between the patients with and without HCC.

\begin{tabular}{|c|c|c|c|c|c|c|}
\hline Biomarker & AUC, $95 \% C I$ & Cut-off * & Se & Sp & $+\mathrm{LR}$ & $-\mathbf{L R}$ \\
\hline AFP (ng/mL) & $0.763,0.696-0.821$ & $>4.4$ & 76.4 & 68.9 & 2.46 & 0.34 \\
\hline $\begin{array}{c}\text { PIVKA-II } \\
\text { (mAU/mL) }\end{array}$ & $0.853,0.794-0.900$ & $>56$ & 75.0 & 85.7 & 5.25 & 0.29 \\
\hline GPC-3 (pg/mL) & $0.759,0.691-0.817$ & $>64$ & 62.5 & 82.4 & 3.54 & 0.46 \\
\hline $\begin{array}{l}\text { Adiponectin } \\
(\mu \mathrm{g} / \mathrm{mL})\end{array}$ & $0.770,0.704-0.828$ & $>3.68$ & 62.5 & 81.5 & 3.38 & 0.46 \\
\hline IL-6 (pg/mL) & $0.731,0.662-0.792$ & $>3.6$ & 79.2 & 62.2 & 2.09 & 0.34 \\
\hline
\end{tabular}

${ }^{*}$ Identified by the Youden $J$ statistic. AUC values were calculated by the receiver operating characteristic curve analysis. Abbreviations: alpha-fetoprotein (AFP), area under the curve (AUC), confidence interval (CI), glypican-3 (GPC-3), hepatocellular carcinoma (HCC), interleukin-6 (IL-6), protein induced by vitamin K absence or antagonist II (PIVKA-II), sensitivity (Se), specificity (Sp), positive likelihood ratio (+LR) and negative likelihood ratio (-LR). 


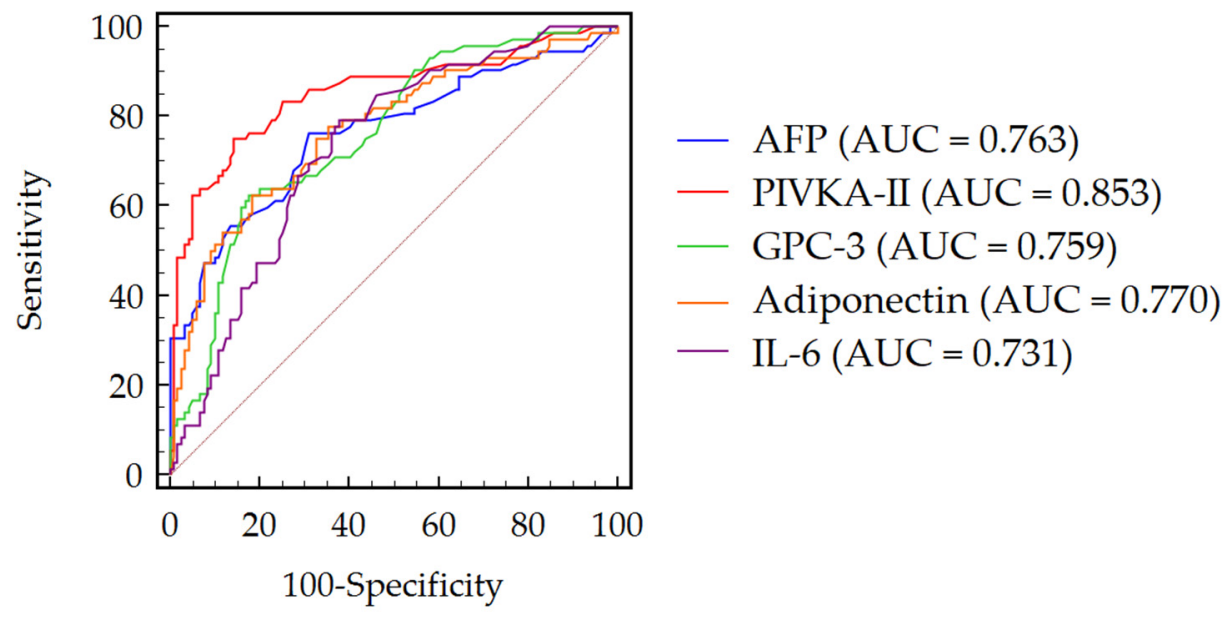

Figure 3. ROC curves of AFP, PIVKA-II, GPC-3, adiponectin and IL-6 discrimination between patients with and without HCC. Abbreviations: alpha-fetoprotein (AFP), area under the curve (AUC), glypican 3 (GPC-3), interleukin-6 (IL-6) and protein induced by vitamin K absence or antagonist II (PIVKA-II).

\subsection{Predictors of HCC and Model Development}

Since the two groups of patients (i.e., patients with and without HCC) showed significant differences regarding the demographic, biochemical and clinical features, we performed a multivariate logistic regression analysis to assess the strength of the association with HCC. Age, gender, BMI, ALT, $\gamma \mathrm{GT}$, platelet count, albumin, total bilirubin, INR, cholesterol, triglycerides, AFP, PIVKA-II, GPC-3, adiponectin and IL-6 were considered for inclusion in the multivariate analysis. A logistic regression analysis was based on a stepwise approach keeping the variables at a significance level below 0.01 [28]. A Log transformation was made to AFP, PIVKA-II, GPC-3 and IL-6 due to data skewness. The variables retained in the model are reported in Table 4.

Table 4. Multivariate analysis for the factors associated to HCC.

\begin{tabular}{ccc}
\hline Variables & OR, 95\% CI & $p$-Value \\
\hline Age (years) & $1.09(1.02-1.16)$ & 0.007 \\
Male gender & $11.95(3.48-41.04)$ & $<0.001$ \\
PIVKA-II (Log mAU $/ \mathrm{mL})$ & $6.87(2.03-23.23)$ & 0.002 \\
GPC-3 (Log pg/mL) & $15.63(2.99-81.59)$ & 0.001 \\
Adiponectin $(\mu \mathrm{g} / \mathrm{mL})$ & $1.54(1.23-1.94)$ & $<0.001$
\end{tabular}

Abbreviations: confidence interval (CI), glypican-3 (GPC-3), hepatocellular carcinoma (HCC), odds ratio (OR) and protein induced by vitamin $\mathrm{K}$ absence or antagonist II (PIVKA-II).

The obtained formula of the model was:

$\mathrm{y}=-17.33+0.09 \times$ Age $+2.48 \times$ Gender $+1.93 \times$ Log PIVKA-II $+2.75 \times$ Log GPC-3 + $0.43 \times$ adiponectin

where age in years, 1 for males and 0 for females; the probability $(p)$ of HCC is given by:

$$
\mathrm{p}=1 /\left(1+\mathrm{e}^{-\mathrm{y}}\right) \text {. }
$$

The median pHCC values were 5.0\% (95\% CI 3.5-8.3) and 82.2\% (95\% CI 75.0-96.5) in patients with and without HCC, respectively $(p<0.001)$. The model showed an excellent diagnostic accuracy for the detection of HCC (AUC $=0.948)$, with a percentage of the patients correctly classified as $87 \%$ in the cross-validation (Figure 4 ). At the cut-off pHCC $=50 \%$, the model showed $\mathrm{Sp}=88.1 \%$, $\mathrm{Se}=86.9 \%,+\mathrm{LR}=9.00$ and $-\mathrm{LR}=0.15$ for the detection of HCC. 
A

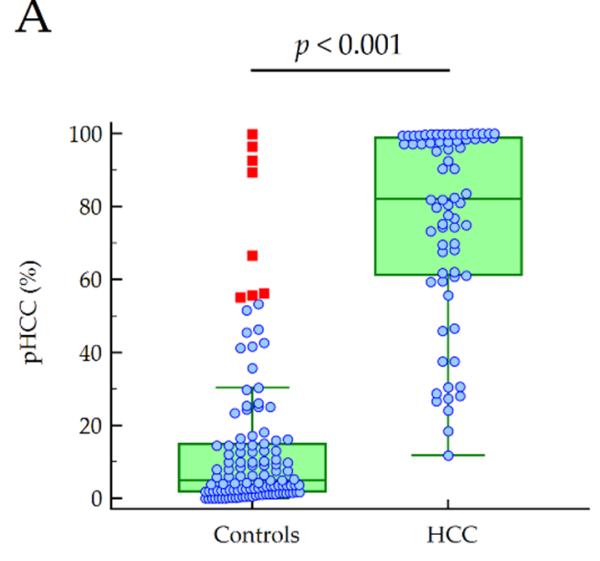

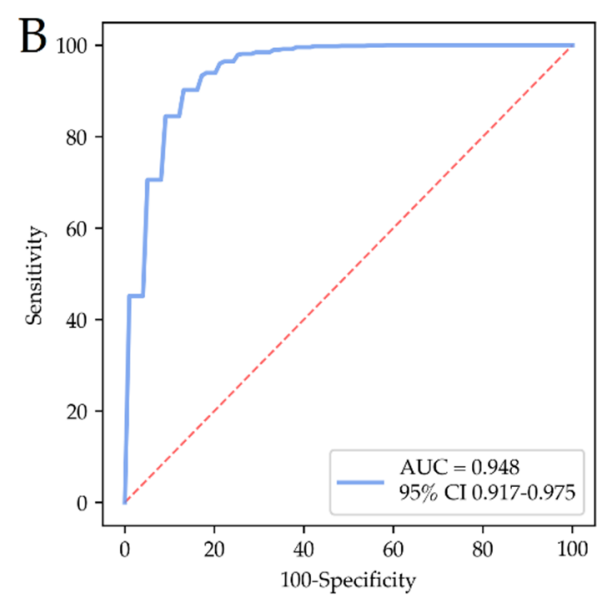

Figure 4. Median values of $\mathrm{pHCC}$ in patients with and without HCC (A) and diagnostic accuracy of the model (B). Red squares indicate values that are larger than the upper quartile plus 3 times the interquartile range. $p$-values were calculated by the Mann-Whitney test. Abbreviations: area under the curve (AUC), confidence interval (CI), hepatocellular carcinoma (HCC) and probability of hepatocellular carcinoma ( $\mathrm{pHCC})$.

\section{Discussion}

In the present study, we investigated the performances of different biomarkers involved in the oncogenic mechanisms of HCC in patients with NAFLD. Indeed, the biomarkers studied were selected on the premise that tumor development in such patients is driven by the concurrent activation of different oncogenic signaling pathways in accordance with the "multiple hits hypothesis" [29,30], whereby comorbidities, genetic determinants and environmental factors simultaneously contribute to NAFLD/NASH-HCC progression [31]. Interestingly, we observed that circulating biomarkers such as PIVKA-II and adiponectin displayed a good performance for the discrimination between patients with HCC and those without tumors; remarkably, the combination of demographic features (i.e., age and gender) together with oncogenic markers (i.e., PIVKA-II and GPC-3) and markers of adipose tissue dysfunction (i.e., adiponectin) allowed the development of a model showing an excellent performance for the detection of HCC.

Several different biomarkers have been studied in the last decades in order to improve and even personalize the surveillance of patients at high risk for HCC development [32-34]. Promising results have derived from comprehensive approaches that have allowed the detection of a wide spectrum of circulating molecules, including tumor proteins and nucleic acids (i.e., circulating tumor DNA/RNA) derived from the primary tumor [35], epigenetic biomarkers such as DNA methylation profiles and noncoding RNAs [36,37] and genetic variants recapitulated in polygenic risk scores [38]. On the other hand, the study of serologic biomarkers such as AFP and PIVKA-II has been pursued over time due to their inexpensiveness, analytical standardization, and acceptable performances [17].

In agreement with previous studies performed in patients with NAFLD $[39,40]$, we observed a good diagnostic accuracy for PIVKA-II (AUC $=0.853$ ) and a moderate performance for AFP (AUC $=0.763)$. Interestingly, GPC-3 showed a higher accuracy compared to the results of our previous study carried out on a cohort of patients with viral related-HCC [18]. Possibly, both the diverse etiology and clinical characteristics of the patients included may have accounted for the discrepancy observed. In particular, the different prevalence of cirrhosis in the control groups could have affected the performance of GPC-3. Indeed, cirrhosis is a preneoplastic condition characterized by genetic, epigenetic and molecular alterations not yet established in patients with chronic hepatitis but frequently observed in HCC [41-43]. As a matter of fact, the more we reduce the clinical differences between two groups of patients, the more the performance of the biomarker decreases. 
Noteworthy, adiponectin showed a performance similar to AFP for the detection of HCC. A recent meta-analysis including nine studies and a total of 705 HCC patients and 1390 healthy controls showed that higher adiponectin levels were significantly associated with liver cancer (standard mean difference $=0.97,95 \%$ CI 0.02 1.93, $p<0.05$ ) [44]. Furthermore, in patients with HCV-related cirrhosis, higher serum adiponectin levels resulted in a predictor of HCC development [45,46] and liver-related mortality [47]. However, the mechanism by which adiponectin is involved in HCC development is not fully clear. Reduced adiponectin levels have been associated with metabolic syndrome [4,48]; nevertheless, studies in vitro and in the animal model have revealed an antiproliferative activity for adiponectin $[49,50]$, suggesting that increased adiponectin levels might have a protective role in HCC. Consonant with this hypothesis, the administration of adiponectin $(5 \mu \mathrm{g} / \mathrm{kg}$ weekly) blocked tumor progression in a thioacetamide-induced rat HCC model, resulting in an $80 \%$ increased survival rate, $73 \%$ reduced average number of nodules and $46 \%$ decreased serum AFP [51]. Further studies are needed to confirm the hepatoprotective and chemoprotective effects of adiponectin against HCC.

Finally, the combination of different biomarkers with clinical and/or demographic characteristics into simple prediction models allowed the further improvement of the diagnostic accuracy for HCC detection [52]. However, the majority of these models have been developed and validated in cohorts of patients chronically infected with HBV or HCV [28,53-55]; to the best of our knowledge, only the GALAD score was tested in the setting of NAFLD, showing a high performance for the identification of patients with HCC [56]. Here, we developed a model including age, gender, PIVKA-II, GPC-3 and adiponectin that showed a high diagnostic accuracy in cross-validation (AUC $=0.948$ ) for the detection of HCC in patients with dysmetabolic liver disease; the model allowed to correctly identify $87 \%$ of the patients included in the study. Given the relatively small number of patients enrolled and the lack of a validation cohort, we applied a machine learning approach based on a stratified cross-validation to assess the performance of the model; accordingly, the original sample was partitioned into a training set to train the model and a test set to evaluate its performance, and the procedure was repeated multiple times. As a result, the model denoted a high accuracy, with a low risk of overfitting, and a generalizability of the independent datasets. However, further multicenter studies are needed to independently validate these findings. Furthermore, a cost-effective analysis may be useful to assess the benefits produced by the implementation of the model in the clinical setting with respect to its cost.

\section{Conclusions}

Our data confirmed the good diagnostic accuracy of PIVKA-II for the detection of HCC in patients with NAFLD. Furthermore, the combination of age, gender, PIVKA-II, GPC-3 and adiponectin allowed a noticeable improvement in the detection of HCC compared to each single biomarker used alone. These findings need to be validated in a prospective surveillance setting in order to assess the ability of the model to predict the HCC occurrence in patients with dysmetabolic liver disease at risk of tumor development.

Supplementary Materials: The following are available online at https:/ / www.mdpi.com/article / 10.3390/cancers13102305/s1: Figure S1: Median values of AFP (A), PIVKA-II (B), GPC-3 (C), adiponectin (D), leptin (E) and IL-6 (F), according to the different stages of liver disease, Figure S2. Diagnostic accuracy of AFP, PIVKA-II, GPC-3, adiponectin and IL-6 for the detection of HCC in lean (A) and obese patients (B), and in normal-glucose tolerant (C) and diabetic patients (D), Figure S3. Diagnostic accuracy of AFP, PIVKA-II, GPC-3, adiponectin and IL-6 for the detection of early HCC, Table S1: Median biomarker values according to the different stages of liver disease, Table S2: Comparison of the diagnostic accuracy of AFP, PIVKA-II, GPC-3, adiponectin and IL-6 for the detection of HCC. 
Author Contributions: Conceptualization, G.P.C.; methodology, G.P.C., S.G. and E.B.; software, G.P.C., A.A., C.R. and D.G.R.; formal analysis, G.P.C. and S.A.; investigation, G.P.C., A.A., C.R., S.G., E.R., M.L.A., A.O., A.N., M.G., P.C., G.M.S. and E.B.; resources, G.P.C., M.L.A. and E.B.; data curation, G.P.C., A.A., C.R., S.G., E.R. and P.C.; writing-original draft preparation, G.P.C.; writing-review and editing, S.G., G.M.S. and E.B.; visualization, G.P.C., S.A. and D.G.R.; supervision, S.G., G.M.S. and E.B. and funding acquisition, M.L.A. and E.B. All authors have read and agreed to the published version of the manuscript.

Funding: This research was funded by the Italian Ministry of Health, grant RF-2016-02364358 (Ricerca Finalizzata, Ministero della Salute), by the Italian Ministry for Education, University and Research (Ministero dell'Istruzione, dell'Università e della Ricerca-MIUR) under the program “Dipartimenti di Eccellenza 2018-2022", project code D15D18000410001, and by a University of Turin local research grant, \#ABAM_RILO_20_01.

Institutional Review Board Statement: This study was conducted according to the guidelines of the Declaration of Helsinki and approved by the Institutional Ethics Committee of A.O.U. Città della Salute e della Scienza di Torino (CEI-452; 6 June 2012).

Informed Consent Statement: Written informed consent was obtained from all subjects involved in the study.

Data Availability Statement: The data presented in this study are available upon request from the corresponding author.

Acknowledgments: The authors thank Roland Andersson (Fujirebio Diagnostics AB) for providing the GPC-3 assays.

Conflicts of Interest: G.P.C. received research grants from Fujirebio Europe and Fujirebio Diagnostics AB. All the other authors declare no conflicts of interest. Fujirebio Diagnostics AB had no role in the design of the study; in the collection, analyses or interpretation of the data; in the writing of the manuscript or in the decision to publish the results.

\section{References}

1. Younossi, Z.M.; Koenig, A.B.; Abdelatif, D.; Fazel, Y.; Henry, L.; Wymer, M. Global epidemiology of nonalcoholic fatty liver disease-Meta-analytic assessment of prevalence, incidence, and outcomes. Hepatology 2016, 64, 73-84. [CrossRef]

2. Marengo, A.; Rosso, C.; Bugianesi, E. Liver Cancer: Connections with Obesity, Fatty Liver, and Cirrhosis. Annu. Rev. Med. 2016, 67, 103-117. [CrossRef]

3. Younossi, Z.; Tacke, F.; Arrese, M.; Chander Sharma, B.; Mostafa, I.; Bugianesi, E.; Wai-Sun Wong, V.; Yilmaz, Y.; George, J.; Fan, J.; et al. Global Perspectives on Nonalcoholic Fatty Liver Disease and Nonalcoholic Steatohepatitis. Hepatology 2019, 69, 2672-2682. [CrossRef]

4. Rajesh, Y.; Sarkar, D. Association of Adipose Tissue and Adipokines with Development of Obesity-Induced Liver Cancer. Int. J. Mol. Sci. 2021, 22, 2163. [CrossRef]

5. Perego, S.; Sansoni, V.; Ziemann, E.; Lombardi, G. Another Weapon against Cancer and Metastasis: Physical-Activity-Dependent Effects on Adiposity and Adipokines. Int. J. Mol. Sci. 2021, 22, 2005. [CrossRef] [PubMed]

6. Shu, R.Z.; Zhang, F.; Wang, F.; Feng, D.C.; Li, X.H.; Ren, W.H.; Wu, X.L.; Yang, X.; Liao, X.D.; Huang, L.; et al. Adiponectin deficiency impairs liver regeneration through attenuating STAT3 phosphorylation in mice. Lab. Invest. 2009, 89, 1043-1052. [CrossRef] [PubMed]

7. Park, E.J.; Lee, J.H.; Yu, G.Y.; He, G.; Ali, S.R.; Holzer, R.G.; Osterreicher, C.H.; Takahashi, H.; Karin, M. Dietary and genetic obesity promote liver inflammation and tumorigenesis by enhancing IL-6 and TNF expression. Cell 2010, 140, 197-208. [CrossRef] [PubMed]

8. Lokau, J.; Schoeder, V.; Haybaeck, J.; Garbers, C. Jak-Stat Signaling Induced by Interleukin-6 Family Cytokines in Hepatocellular Carcinoma. Cancers (Basel) 2019, 11, 1704. [CrossRef]

9. Johnson, D.E.; O'Keefe, R.A.; Grandis, J.R. Targeting the IL-6/JAK/STAT3 signalling axis in cancer. Nat. Rev. Clin. Oncol. 2018, 15, 234-248. [CrossRef]

10. Villanueva, A. Hepatocellular Carcinoma. N. Engl. J. Med. 2019, 380, 1450-1462. [CrossRef]

11. European Association for the Study of the Liver. EASL Clinical Practice Guidelines: Management of hepatocellular carcinoma. J. Hepatol. 2018, 69, 182-236. [CrossRef]

12. Marrero, J.A.; Kulik, L.M.; Sirlin, C.B.; Zhu, A.X.; Finn, R.S.; Abecassis, M.M.; Roberts, L.R.; Heimbach, J.K. Diagnosis, Staging, and Management of Hepatocellular Carcinoma: 2018 Practice Guidance by the American Association for the Study of Liver Diseases. Hepatology 2018, 68, 723-750. [CrossRef] [PubMed] 
13. Omata, M.; Cheng, A.L.; Kokudo, N.; Kudo, M.; Lee, J.M.; Jia, J.; Tateishi, R.; Han, K.H.; Chawla, Y.K.; Shiina, S.; et al. AsiaPacific clinical practice guidelines on the management of hepatocellular carcinoma: A 2017 update. Hepatol. Int. 2017, 11, 317-370. [CrossRef] [PubMed]

14. Singal, A.; Volk, M.L.; Waljee, A.; Salgia, R.; Higgins, P.; Rogers, M.A.; Marrero, J.A. Meta-analysis: Surveillance with ultrasound for early-stage hepatocellular carcinoma in patients with cirrhosis. Aliment. Pharmacol. Ther. 2009, 30, 37-47. [CrossRef]

15. Campion, D.; Tucci, A.; Ponzo, P.; Caviglia, G.P. Non-invasive biomarkers for the detection of hepatocellular carcinoma. Minerva Biotecnol. 2019, 31, 11-22. [CrossRef]

16. Huang, D.Q.; El-Serag, H.B.; Loomba, R. Global epidemiology of NAFLD-related HCC: Trends, predictions, risk factors and prevention. Nat. Rev. Gastroenterol. Hepatol. 2020. [CrossRef]

17. Caviglia, G.P.; Ribaldone, D.G.; Abate, M.L.; Ciancio, A.; Pellicano, R.; Smedile, A.; Saracco, G.M. Performance of protein induced by vitamin $\mathrm{K}$ absence or antagonist-II assessed by chemiluminescence enzyme immunoassay for hepatocellular carcinoma detection: A meta-analysis. Scand. J. Gastroenterol. 2018, 53, 734-740. [CrossRef]

18. Caviglia, G.P.; Ciruolo, M.; Abate, M.L.; Carucci, P.; Rolle, E.; Rosso, C.; Olivero, A.; Troshina, G.; Risso, A.; Nicolosi, A.; et al. Alpha-Fetoprotein, Protein Induced by Vitamin K Absence or Antagonist II and Glypican-3 for the Detection and Prediction of Hepatocellular Carcinoma in Patients with Cirrhosis of Viral Etiology. Cancers (Basel) 2020, 12, 3218. [CrossRef] [PubMed]

19. Loglio, A.; Iavarone, M.; Facchetti, F.; Di Paolo, D.; Perbellini, R.; Lunghi, G.; Ceriotti, F.; Galli, C.; Sandri, M.T.; Viganò, M.; et al. The combination of PIVKA-II and AFP improves the detection accuracy for HCC in HBV caucasian cirrhotics on long-term oral therapy. Liver Int. 2020, 40, 1987-1996. [CrossRef]

20. Ricco, G.; Cosma, C.; Bedogni, G.; Biasiolo, A.; Guarino, M.; Pontisso, P.; Morisco, F.; Oliveri, F.; Cavallone, D.; Bonino, F.; et al. Modeling the time-related fluctuations of AFP and PIVKA-II serum levels in patients with cirrhosis undergoing surveillance for hepatocellular carcinoma. Cancer Biomark. 2020, 29, 189-196. [CrossRef] [PubMed]

21. Hu, D.; Su, C.; Sun, L.; Gao, Y.; Li, Y. Performance of Serum Glypican 3 in Diagnosis of Hepatocellular Carcinoma: A meta-analysis. Ann Hepatol. 2019, 18, 58-67.

22. Younossi, Z.; Stepanova, M.; Sanyal, A.J.; Harrison, S.A.; Ratziu, V.; Abdelmalek, M.F.; Diehl, A.M.; Caldwell, S.; Shiffman, M.L.; Schall, R.A.; et al. The conundrum of cryptogenic cirrhosis: Adverse outcomes without treatment options. J. Hepatol. 2018, 69, 1365-1370. [CrossRef] [PubMed]

23. Boyle, M.; Masson, S.; Anstee, Q.M. The bidirectional impacts of alcohol consumption and the metabolic syndrome: Cofactors for progressive fatty liver disease. J. Hepatol. 2018, 68, 251-267. [CrossRef] [PubMed]

24. O'Shea, R.S.; Dasarathy, S.; McCullough, A.J. Alcoholic liver disease. Hepatology 2010, 51, 307-328. [CrossRef]

25. Kleiner, D.E.; Brunt, E.M.; Van Natta, M.; Behling, C.; Contos, M.J.; Cummings, O.W.; Ferrel, L.D.; Liu, Y.C.; Torbenson, M.S.; Unalp-Arida, A.; et al. Design and validation of a histological scoring system for nonalcoholic fatty liver disease. Hepatology 2005, 41, 1313-1321. [CrossRef] [PubMed]

26. Caviglia, G.P.; Touscoz, G.A.; Smedile, A.; Pellicano, R. Noninvasive assessment of liver fibrosis: Key messages for clinicians. Pol. Arch. Med. Wewn. 2014, 124, 329-335. [CrossRef] [PubMed]

27. Gaia, S.; Campion, D.; Evangelista, A.; Spandre, M.; Cosso, L.; Brunello, F.; Ciccone, G.; Bugianesi, E.; Rizzetto, M. Non-invasive score system for fibrosis in chronic hepatitis: Proposal for a model based on biochemical, FibroScan and ultrasound data. Liver Int. 2015, 35, 2027-2035. [CrossRef]

28. Johnson, P.J.; Pirrie, S.J.; Cox, T.F.; Berhane, S.; Teng, M.; Palmer, D.; Morse, J.; Hull, D.; Patman, G.; Kagebayashi, C.; et al. The detection of hepatocellular carcinoma using a prospectively developed and validated model based on serological biomarkers. Cancer Epidemiol. Biomarkers Prev. 2014, 23, 144-153. [CrossRef]

29. Takakura, K.; Oikawa, T.; Nakano, M.; Saeki, C.; Torisu, Y.; Kajihara, M.; Saruta, M. Recent Insights Into the Multiple Pathways Driving Non-alcoholic Steatohepatitis-Derived Hepatocellular Carcinoma. Front. Oncol. 2019, 9, 762. [CrossRef]

30. Raza, S.; Rajak, S.; Anjum, B.; Sinha, R.A. Molecular links between non-alcoholic fatty liver disease and hepatocellular carcinoma. Hepatoma Res. 2019, 5, 42. [CrossRef]

31. Drescher, H.K.; Weiskirchen, S.; Weiskirchen, R. Current Status in Testing for Nonalcoholic Fatty Liver Disease (NAFLD) and Nonalcoholic Steatohepatitis (NASH). Cells 2019, 8, 845. [CrossRef] [PubMed]

32. Piñero, F.; Dirchwolf, M.; Pessôa, M.G. Biomarkers in Hepatocellular Carcinoma: Diagnosis, Prognosis and Treatment Response Assessment. Cells 2020, 9, 1370. [CrossRef] [PubMed]

33. Pan, Y.; Chen, H.; Yu, J. Biomarkers in Hepatocellular Carcinoma: Current Status and Future Perspectives. Biomedicines 2020, 8, 576. [CrossRef] [PubMed]

34. Caviglia, G.P.; Abate, M.L.; Gaia, S.; Petrini, E.; Bosco, C.; Olivero, A.; Rosso, C.; Ciancio, A.; Pellicano, R.; Saracco, G.M.; et al. Risk of hepatocellular carcinoma in HBV cirrhotic patients assessed by the combination of miR-122, AFP and PIVKA-II. Panminerva Med. 2017, 59, 283-289.

35. Mocan, T.; Simão, A.L.; Castro, R.E.; Rodrigues, C.M.P.; Słomka, A.; Wang, B.; Strassburg, C.; Wöhler, A.; Willms, A.G.; Kornek, M. Liquid Biopsies in Hepatocellular Carcinoma: Are We Winning? J. Clin. Med. 2020, 9, 1541. [CrossRef]

36. Arechederra, M.; Recalde, M.; Gárate-Rascón, M.; Fernández-Barrena, M.G.; Ávila, M.A.; Berasain, C. Epigenetic Biomarkers for the Diagnosis and Treatment of Liver Disease. Cancers 2021, 13, 1265. [CrossRef] [PubMed]

37. Petrini, E.; Caviglia, G.P.; Abate, M.L.; Fagoonee, S.; Smedile, A.; Pellicano, R. MicroRNAs in HBV-related hepatocellular carcinoma: Functions and potential clinical applications. Panminerva Med. 2015, 57, 201-209. [PubMed] 
38. Bianco, C.; Jamialahmadi, O.; Pelusi, S.; Baselli, G.; Dongiovanni, P.; Zanoni, I.; Santoro, L.; Maier, S.; Liguori, A.; Meroni, M.; et al. Non-invasive stratification of hepatocellular carcinoma risk in non-alcoholic fatty liver using polygenic risk scores. J. Hepatol. 2021, 74, 775-782. [CrossRef] [PubMed]

39. Beale, G.; Chattopadhyay, D.; Gray, J.; Stewart, S.; Hudson, M.; Day, C.; Trerotoli, P.; Giannelli, G.; Manas, D.; Reeves, H. AFP, PIVKAII, GP3, SCCA-1 and follisatin as surveillance biomarkers for hepatocellular cancer in non-alcoholic and alcoholic fatty liver disease. BMC Cancer 2008, 8, 200. [CrossRef] [PubMed]

40. Ricco, G.; Cavallone, D.; Cosma, C.; Caviglia, G.P.; Oliveri, F.; Biasiolo, A.; Abate, M.L.; Plebani, M.; Smedile, A.; Bonino, F.; et al. Impact of etiology of chronic liver disease on hepatocellular carcinoma biomarkers. Cancer Biomark. 2018, 21, 603-612. [CrossRef] [PubMed]

41. Dhanasekaran, R.; Bandoh, S.; Roberts, L.R. Molecular pathogenesis of hepatocellular carcinoma and impact of therapeutic advances. F1000Res. 2016, 5. F1000 Faculty Rev-879. [CrossRef]

42. Kanda, T.; Goto, T.; Hirotsu, Y.; Moriyama, M.; Omata, M. Molecular Mechanisms Driving Progression of Liver Cirrhosis towards Hepatocellular Carcinoma in Chronic Hepatitis B and C Infections: A Review. Int. J. Mol. Sci. 2019, 20, 1358. [CrossRef]

43. Caviglia, G.P.; Abate, M.L.; Rolle, E.; Carucci, P.; Armandi, A.; Rosso, C.; Olivero, A.; Ribaldone, D.G.; Tandoi, F.; Saracco, G.M.; et al. The Clinical Role of Serum Epidermal Growth Factor Receptor 3 in Hepatitis C Virus-Infected Patients with Early Hepatocellular Carcinoma. Biology 2021, 10, 215. [CrossRef] [PubMed]

44. Song, R.R.; Gu, X.L. Serum adiponectin levels may be associated with the pathogenesis of hepatocellular carcinoma. Tumour Biol. 2015, 36, 2983-2992. [CrossRef] [PubMed]

45. Arano, T.; Nakagawa, H.; Tateishi, R.; Ikeda, H.; Uchino, K.; Enooku, K.; Goto, E.; Masuzaki, R.; Asaoka, Y.; Kondo, Y.; et al. Serum level of adiponectin and the risk of liver cancer development in chronic hepatitis C patients. Int. J. Cancer 2011, 129, 2226-2235. [CrossRef]

46. Bastard, J.P.; Fellahi, S.; Audureau, É; Layese, R.; Roudot-Thoraval, F.; Cagnot, C.; Mahuas-Bourcier, V.; Sutton, A.; Ziol, M.; Capeau, J.; et al. Elevated adiponectin and sTNFRII serum levels can predict progression to hepatocellular carcinoma in patients with compensated HCV1 cirrhosis. Eur. Cytokine Netw. 2018, 29, 112-120. [CrossRef]

47. Nakagawa, H.; Fujiwara, N.; Tateishi, R.; Arano, T.; Nakagomi, R.; Kondo, M.; Minami, T.; Sato, M.; Uchino, K.; Enooku, K.; et al. Impact of serum levels of interleukin-6 and adiponectin on all-cause, liver-related, and liver-unrelated mortality in chronic hepatitis C patients. J. Gastroenterol. Hepatol. 2015, 30, 379-388. [CrossRef]

48. Bugianesi, E.; Pagotto, U.; Manini, R.; Vanni, E.; Gastaldelli, A.; de Iasio, R.; Gentilcore, E.; Natale, S.; Cassader, M.; Rizzetto, M.; et al. Plasma adiponectin in nonalcoholic fatty liver is related to hepatic insulin resistance and hepatic fat content, not to liver disease severity. J. Clin. Endocrinol. Metab. 2005, 90, 3498-3504. [CrossRef] [PubMed]

49. Saxena, N.K.; Fu, P.P.; Nagalingam, A.; Wang, J.; Handy, J.; Cohen, C.; Tighiouart, M.; Sharma, D.; Anania, F.A. Adiponectin modulates $\mathrm{C}$-jun $\mathrm{N}$-terminal kinase and mammalian target of rapamycin and inhibits hepatocellular carcinoma. Gastroenterology 2010, 139, 1762-1773. [CrossRef]

50. Man, K.; Ng, K.T.; Xu, A.; Cheng, Q.; Lo, C.M.; Xiao, J.W.; Sun, B.S.; Lim, Z.X.; Cheung, J.S.; Wu, E.X.; et al. Suppression of liver tumor growth and metastasis by adiponectin in nude mice through inhibition of tumor angiogenesis and downregulation of Rho kinase/IFN-inducible protein 10/matrix metalloproteinase 9 signaling. Clin. Cancer Res. 2010, 16, 967-977. [CrossRef]

51. Al-Gayyar, M.M.; Abbas, A.; Hamdan, A.M. Chemopreventive and hepatoprotective roles of adiponectin (SULF2 inhibitor) in hepatocelluar carcinoma. Biol. Chem. 2016, 397, 257-267. [CrossRef]

52. Sumida, Y.; Yoneda, M.; Seko, Y.; Ishiba, H.; Hara, T.; Toyoda, H.; Yasuda, S.; Kumada, T.; Hayashi, H.; Kobayashi, T.; et al. Surveillance of Hepatocellular Carcinoma in Nonalcoholic Fatty Liver Disease. Diagnostics (Basel) 2020, 10, 579. [CrossRef] [PubMed]

53. Pirisi, M.; Leutner, M.; Romito, R.; Gaia, S. Novel Calculators for Risk Stratification in Hepatocellular Carcinoma: A Concise Review. J. Clin. Gastroenterol. 2020, 54, 789-794. [CrossRef] [PubMed]

54. Caviglia, G.P.; Abate, M.L.; Petrini, E.; Gaia, S.; Rizzetto, M.; Smedile, A. Highly sensitive alpha-fetoprotein, Lens culinaris agglutinin-reactive fraction of alpha-fetoprotein and des-gamma-carboxyprothrombin for hepatocellular carcinoma detection. Hepatol. Res. 2016, 46, E130-E135. [CrossRef] [PubMed]

55. Fan, R.; Papatheodoridis, G.; Sun, J.; Innes, H.; Toyoda, H.; Xie, Q.; Mo, S.; Sypsa, V.; Guha, I.N.; Kumada, T.; et al. aMAP risk score predicts hepatocellular carcinoma development in patients with chronic hepatitis. J. Hepatol. 2020, 73, 1368-1378. [CrossRef]

56. Best, J.; Bechmann, L.P.; Sowa, J.P.; Sydor, S.; Dechêne, A.; Pflanz, K.; Bedreli, S.; Schotten, C.; Geier, A.; Berg, T.; et al. GALAD Score Detects Early Hepatocellular Carcinoma in an International Cohort of Patients With Nonalcoholic Steatohepatitis. Clin. Gastroenterol. Hepatol. 2020, 18, 728-735. [CrossRef] [PubMed] 